\title{
Optoacoustic imaging using thin polymer étalon
}

\author{
Shai Ashkenazi, Yang Hou, Takashi Buma, and Matthew O'Donnell \\ Department of Biomedical Engineering, University of Michigan, Ann Arbor, Michigan 48109-2099
}

(Received 29 November 2004; accepted 14 February 2005; published online 21 March 2005)

\begin{abstract}
Optical detection of ultrasound is a promising technique for high frequency imaging arrays. Detection resolution approaches the optical resolution, which can be on the order of the optical wavelength. We describe here an optical technique for ultrasound detection based on a thin $(10 \mu \mathrm{m})$ Fabry-Perot étalon optimized for high resolution imaging. The signal to noise ratio (SNR) approaches that of an ideal piezoelectric transducer over a $100 \mathrm{MHz}$ bandwidth. Array functionality is demonstrated by scanning a probe beam along a line. Thermoelastic excitation was applied to generate acoustic waves in a test phantom containing a single "pointlike" source. An image of the source was reconstructed using signals acquired from the étalon detector array. (c) 2005 American Institute of Physics. [DOI: 10.1063/1.1896085]
\end{abstract}

The use of ultrasonic imaging at high frequencies (acoustic microscopy) in science and industry has greatly increased during the last decade, reaching many new applications. For example, the microelectronics industry applies ultrasonic imaging at frequencies in the range of $100-200 \mathrm{MHz}$ to inspect IC packaging ${ }^{1}$ and interconnections ${ }^{2}$ and to monitor automated assembly processes such as flip-chip technology. ${ }^{3}$ Acoustic microscopy also is a valuable imaging tool for developmental biology since it allows noninvasive imaging of mice from very early embryonic stages $^{4-6}$ through neonate and adulthood. Pharmaceutical research makes extensive use of mice as disease models. Indeed, acoustic microscopy is nearly ideal for noninvasive longitudinal studies of disease progression and regression. ${ }^{7,8}$ In these applications the useful frequency range is $20-60 \mathrm{MHz}$, where image resolution is typically $60 \mu \mathrm{m}$ at a penetration depth of $8 \mathrm{~mm} .{ }^{9}$ Medical applications of acoustic microscopy include imaging of internal structures of the eye, ${ }^{10,11}$ diagnosing skin pathologies, ${ }^{12}$ and intravascular imaging (IVUS).

As opposed to ultrasonic imaging in the low $\mathrm{MHz}$ range where piezoelectric array transducers are used, high frequency acoustic microscopy relies on mechanical scanning of a single element transducer. This limits the ultimate quality of real-time imaging. The state of the art highest frequency commercial array transducer is used in IVUS systems and operates at $20 \mathrm{MHz}$. Array transducers at higher frequencies are not available for routine use due to the limitations of piezoelectric array fabrication technology. Major difficulties are encountered in dicing piezoceramics to micron scale elements. Cabling and interconnections pose another serious challenge since each element requires separate frontend electronics to overcome the high element impedance. Developing new transducer technologies to overcome these difficulties would be highly valuable for a wide range of acoustic microscopy applications.

Optical techniques for ultrasound detection have been studied for at least several decades. ${ }^{13-15}$ They typically rely on the interaction of ultrasound strain with the optical field to modulate the optical phase. Optical methods are then applied to demodulate phase information. The most attractive feature of optical detection is the small detection size, easily accomplished using optical focusing. Other attractive features for high resolution imaging are the high bandwidth (typically higher than $100 \mathrm{MHz}$ ) and the ability to form an array using optical imaging.

We have focused on optical detection using a FabryPerot étalon structure as an ultrasound array. ${ }^{15}$ Étalon detection of ultrasound was first demonstrated by Thompson. ${ }^{16}$ More recently, Kostli and Beard ${ }^{17}$ demonstrated a twodimensional étalon array operating at $15 \mathrm{MHz}$. A FabryPerot étalon consists of a transparent layer coated by semitransparent mirrors on both faces. Illuminating the structure with an optical beam creates multiple reflections within the étalon, where optical resonance occurs whenever the round trip path length of the beam within the étalon corresponds to an integer multiple of the optical wavelength. Resonance is characterized by a sharp decrease in the optical reflection near the resonance wavelength. Ultrasonic waves that propagate through the étalon modulate the thickness of the étalon and therefore modulate the optical path length. The intensity of the reflected beam is highly sensitive to the optical path length near resonance. This is the primary mechanism for ultrasound sensitivity. A schematic illustration of this mechanism is given in Fig. 1.

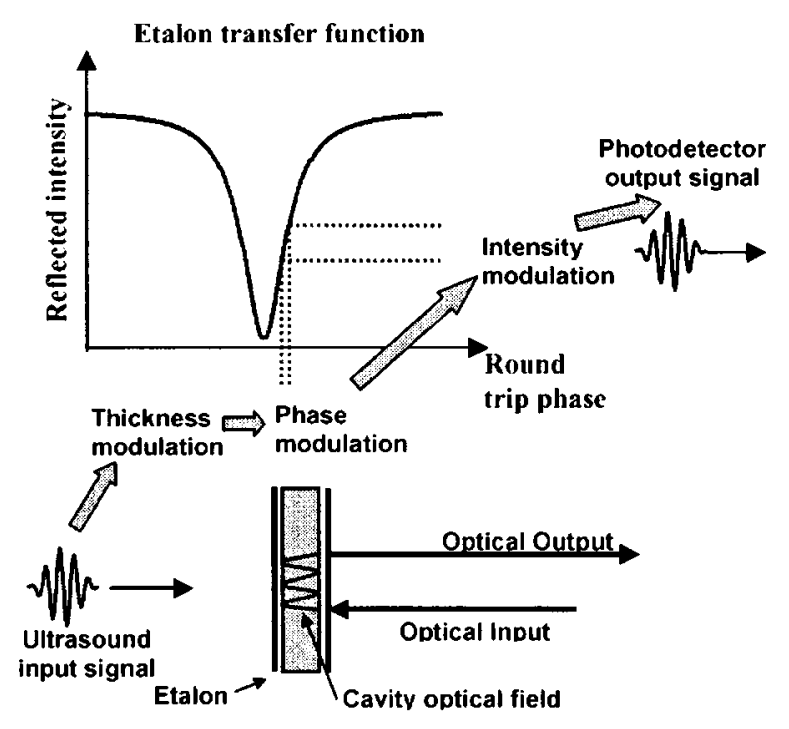

FIG. 1. Schematic illustration of the basic operation principle of the étalon detector. Ultrasound input signal induces thickness modulations of the étalon. The étalon transfer function relates the phase modulations to output intensity modulations. 


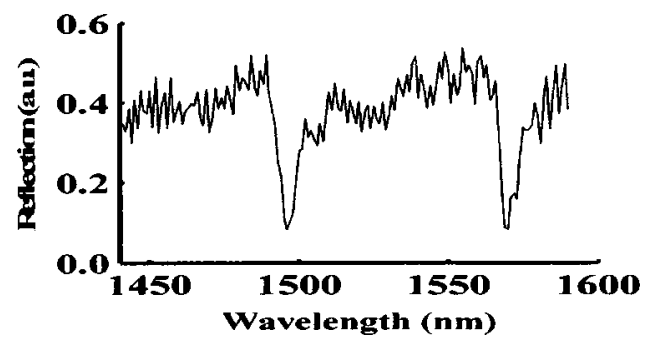

FIG. 2. Spectrum of the reflected intensity of a $10 \mu \mathrm{m}$ thick SU-8 étalon.

We have developed fabrication techniques to produce very thin polymer étalons on a glass substrate. The bulk is formed by spin coating an SU-8 polymer solution (epoxy based photoresist, Microchem Inc.). Vacuum evaporation of thin gold layers is used to form the étalon mirrors. An additional thin polymer layer $(1.5 \mu \mathrm{m})$ is applied as a final step to protect the structure. We chose a polymer as the étalon bulk due to its relatively high compressibility that leads to high sensitivity. Polymer materials are also better matched acoustically to water or tissue environments, thus minimizing acoustic reflections at the étalon's front interface. A glass substrate was used for these initial studies. We plan to construct similar structures on a polymer substrate to better match the entire device to water and tissue environments.

The spatial resolution of an étalon based detector array is limited first by the resolution of the optical system used to image the étalon surface onto a photodetector (either a scanned single photodetector or photodetector array). It can be further degraded because different illumination angles have different optical path lengths within the étalon and therefore different resonance wavelengths. Consequently, different angular components of the focused optical beam will have different detection sensitivity. Since the detection sensitivity will decrease for the higher angular components of the focused beam, the effective $\mathrm{f} / \#$ available for detection is higher than the optical $\mathrm{f} / \#$. ${ }^{18}$ Requiring that the spread of resonance wavelengths due to the angular content of a focused beam is smaller than the resonance width measured at zero angle illumination, yields a limit of $\lambda \sqrt{Q / 8}$ on the highest spatial resolution that can be achieved using étalon detection ( $\lambda$ being the optical wavelength and $Q$ is the quality factor of the resonator defined as the ratio of the resonance frequency to the resonance bandwidth). Consequently, the sensitivity, directly related to the $Q$, must be traded off with element size. We have fabricated a $10 \mu \mathrm{m}$ thick étalon using the technique described above. The relatively small thickness was chosen to support a high temporal bandwidth of $100 \mathrm{MHz}$ and spatial resolution better than $20 \mu \mathrm{m}$. A tunable laser source (HP8168F, HP) in the range of $1440-1590 \mathrm{~nm}$ was used to measure the reflected optical intensity spectrum of the etalon (Fig. 2). Resonance peaks of $Q=300$ were observed. Using the $\lambda \sqrt{Q / 8}$ rule of thumb we estimated a minimal detection size of $9.5 \mu \mathrm{m}$. The sensitivity of the étalon detector was tested by insonifying the étalon with a focused ultrasonic transducer having a center frequency of $50 \mathrm{MHz}$ and an $\mathrm{f} \#$ of 1.4. The tight focusing is achieved using a curved lithium niobate active element. Double quarter wave matching layers optimize acoustic coupling of the active element to water environment, yielding a bandwidth of $60 \% .{ }^{19}$

The optical set-up included a lens focusing the probe beam to a $15 \mu \mathrm{m}$ spot size on the étalon. The reflected light
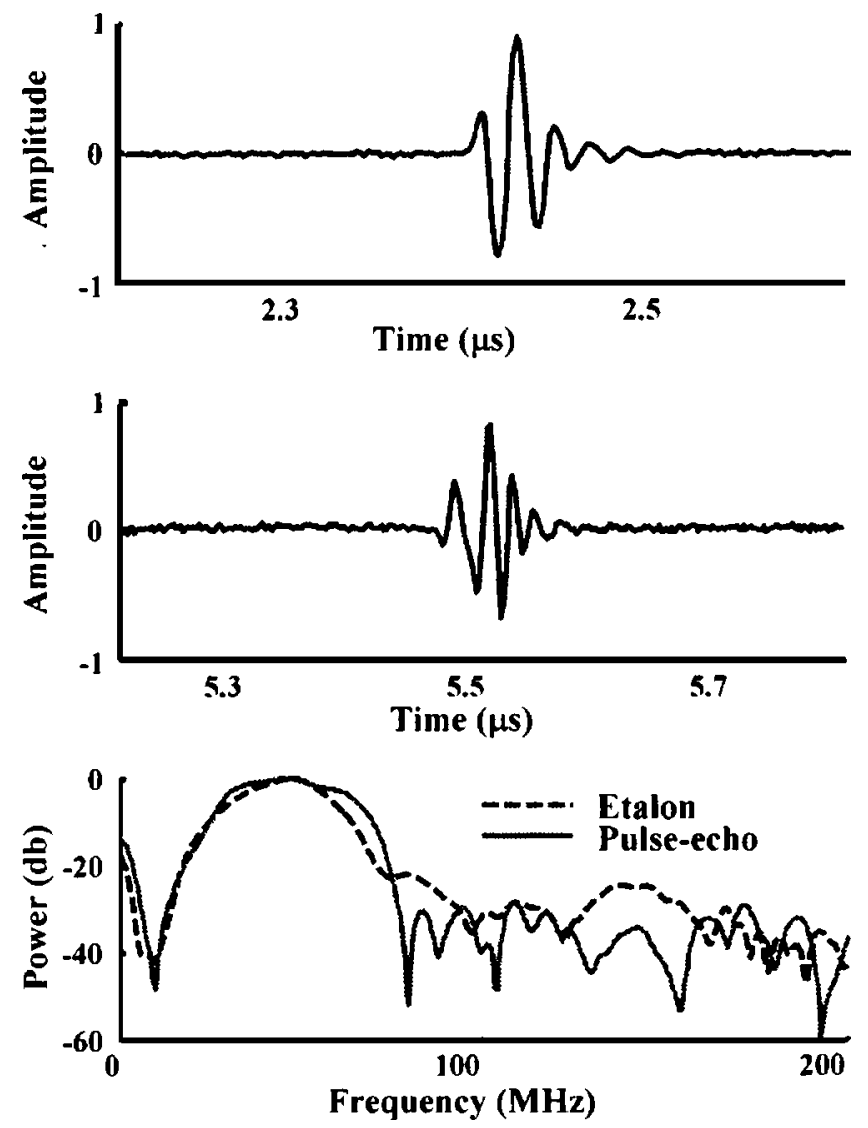

FIG. 3. Signals from the $50 \mathrm{MHz}$ source: (top) étalon signal, (middle) pulse-echo signal, (bottom) power spectra of étalon and pulse-echo signals.

was collected by the same lens and detected using an amplified InGaAs photodiode (818-BB-30A, Newport). The optical reflection signal was recorded and compared with the pulse-echo signal detected by the ultrasonic transducer. Figure 3 shows the étalon signal and the corresponding pulseecho signal (single-shot recordings) in both time and frequency domains. Higher SNR is observed in the étalon signal compared to the pulse-echo. Frequency domain presentation shows enhancement of high frequency components (higher than $50 \mathrm{MHz}$ ) in the étalon response.

The optical set-up was mounted on a precision motorized 2D translation stage to scan the optical probe beam over the étalon surface. Detector array functionality was achieved by recording the étalon signal at multiple positions of the probe beam. To test the imaging capability of the étalon we prepared a gel phantom containing a single black, $50 \mu \mathrm{m}$ diameter, polystyrene sphere. By illuminating the phantom with a pulsed Nd:YAG laser $(\lambda=532 \mathrm{~nm}$, pulse fluence $=8 \mathrm{~mJ} / \mathrm{cm}^{2}$, pulse duration $=4 \mathrm{~ns}$, and repetition rate $=15 \mathrm{~Hz})$ a photoacoustic source was created. Figure 4 shows a typical photoacoustic signal detected by the étalon. The peak acoustic pressure $P_{\text {ac }}$ observed at a distance $r$ from a photoacoustic sphere source of radius $R$ can be expressed as

$$
P_{\mathrm{ac}}=\frac{c^{2} \beta W R}{2 C_{P} r},
$$

where $c$ is the sound velocity, $\beta$ is the thermal expansion coefficient, $W$ is the absorbed energy density, and $C_{P}$ is the specific heat capacity. Using the above expression we estimated the peak acoustic pressure in our case, to be $2.1 \mathrm{kPa}$ at the detection position, representing a noise equivalent pres- 

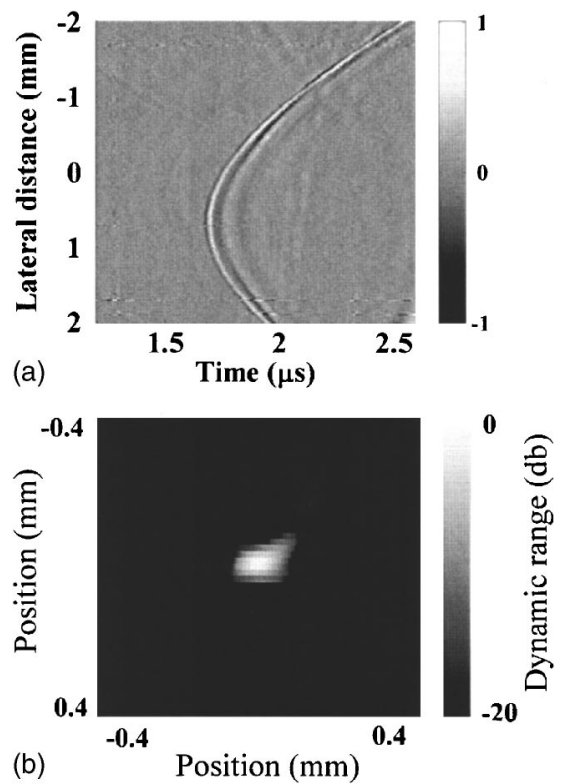

FIG. 4. (Top) Wavefield plot across a $4 \mathrm{~mm}$ imaging aperture of the acoustic field radiated from black $50 \mu \mathrm{m}$ diam sphere after short laser pulse illumination. (Bottom) Reconstructed image of the photoacoustic source (800 $\mu \mathrm{m} \times 800 \mu \mathrm{m}$ field of view, $20 \mathrm{db}$ dynamic range).

sure $(\mathrm{NEP})$ of $0.2 \mathrm{~Pa} / \sqrt{\mathrm{Hz}}$ over a $50 \mathrm{MHz}$ band.

The étalon signal was recorded while scanning the probe beam along a $4 \mathrm{~mm}$ line segment (i.e., imaging aperture) at $20 \mu \mathrm{m}$ intervals. Figure 4 (top) shows a wavefield plot of the detected photoacoustic field. Typical array beam forming with dynamic focusing was applied to these data to produce the image of Fig. 4 (bottom). Bandpass filtering (4 MHz to $40 \mathrm{MHz}$ ) and demodulation schemes were then applied to convert the field image into an intensity image. Clearly, the $50 \mu \mathrm{m}$ diameter photoacoustic source was imaged with high spatial resolution and minimal artifacts.

Development and characterization of a polymeric etalon optical device that functions as an ultrasound detector array has been described. This structure was designed and optimized to detect ultrasound at a very high bandwidth, exceeding $100 \mathrm{MHz}$. Very high spatial resolution is gained by optical focusing of the interrogating laser beam on the etalon that creates an acoustic detection size of approximately $15 \mu \mathrm{m}$.

The optical spot is mechanically scanned to produce an array of detection positions, yet simultaneous detection of more than one array element is highly desirable for real-time imaging. This can be realized by splitting the interrogating beam into several focused spots on the etalon's surface and using a photodiode array to detect the reflected light from each spot. ${ }^{17}$ Two technical issues must be addressed to achieve parallel array detection. First, the laser power should be increased in direct proportion to the number of elements to maintain high SNR. This can be acomplished using Erbium doped fiber amplifiers (EDFA). Each EDFA can support a subarray of 20-30 elements. Second, the étalon should be uniform across the array aperture to minimize differences in sensitivity between array elements. We have tested the uniformity of two types of polymer etalons constructed from

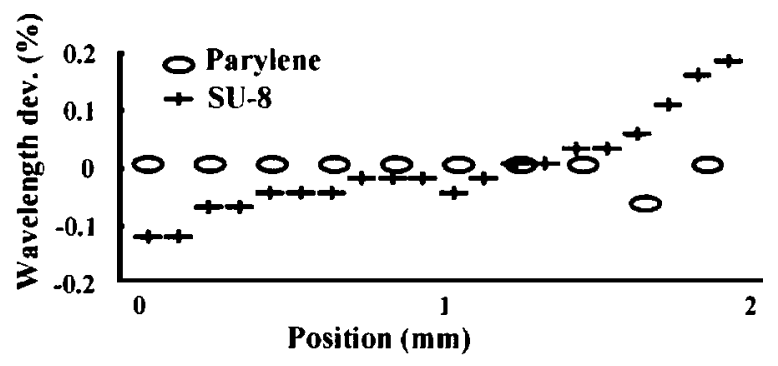

FIG. 5. Relative change in resonance wavelength as a function of position in SU-8 and Parylene étalons

SU8 and Parylene. Parylene etalons are formed using a vacuum deposition polymerization technique. ${ }^{20}$ The change in optical resonance wavelength across a typical array aperture of $2 \mathrm{~mm}$ was measured for the two étalon types (Fig. 5). The uniformity of the Parylene etalon is significantly better than that of the SU8 one, allowing for array interrogation using multiple beams from a single laser source. Combining this result with the high SNR (comparable with that of a large aperture piezoelectric transducer) and very small detection size $(15 \mu \mathrm{m})$ makes the étalon detector a promising alternative to piezoelectric array for high resolution ultrasonic imaging applications.

The authors thank the Resource Center for Medical Ultrasonic Transducer Technology at the University of Southern California for providing the high frequency ultrasonic transducer used in these studies.

${ }^{1}$ J. E. Semmens and L. W. Kessler, "Physical and Failure Analysis of Integrated Circuits," Proceedings of the 10th International Symposium, 2003, p. 103.

${ }^{2}$ J. E. Semmens and L. W. Kessler, Reliability Physics Symposium, 1997, 35th Annual Proceedings., IEEE International, 1997, p. 141.

${ }^{3}$ D. A. Hutt, D. P. Webb, K. C. Hung, C. W. Tang, P. P. Conway, D. C. Whalley, and Y. C. Chan, Electronics Manufacturing Technology Symposium, 2000, Twenty-Sixth IEEE/CPMT International, 2000, p. 191.

${ }^{4}$ A. Liu, A. L. Joyner, and D. H. Turnbull, Mech. Dev. 75, 107 (1998).

${ }^{5}$ S. Srinivasan, H. S. Baldwin, and O. Aristizabal, Circulation 98, 912 (1998).

${ }^{6}$ D. H. Turnbull, Comput. Med. Imaging Graph. 23, 25 (1999).

${ }^{7}$ D. H. Turnbull, J. A. Ramsay, G. S. Shivji, T. S. Bloomfield, L. From, D. N. Sauder, and S. F. Foster, Ultrasound Med. Biol. 22, 845 (1996).

${ }^{8}$ E. D. Goertz, L. J. Yu, S. R. Kerbel, N. P. Burns, and S. F. Foster, Cancer Res. 62, 6371 (2002).

${ }^{9}$ S. F. Foster and M. Y. Zhang, Ultrasound Med. Biol. 28, 1165 (2002).

${ }^{10}$ P. J. Cao, W. H. Chen, N. Karkhanis, and K. K. Shung, Proc. SPIE 4687, 430 (2002).

${ }^{11}$ J. C. Pavlin and S. F. Foster, Ultrasound Biomicroscopy of the Eye (Springer-Verlag, New York, 1995).

${ }^{12}$ M. Vogt, K. Kaspar, P. Altmeyer, K. Hoffmann, and S. El Gammal, Frequenz 55, 12 (2001).

${ }^{13}$ J. P. Monchalin, Appl. Phys. Lett. 47, 14 (1985).

${ }^{14}$ P. C. Beard, F. Perenncs, and T. N. Mills, IEEE Trans. Ultrason. Ferroelectr. Freq. Control 46, 1575 (1999).

${ }^{15}$ J. D. Hamilton and M. O'Donnell, IEEE Trans. Ultrason. Ferroelectr. Freq. Control 45, 216 (1998).

${ }^{16}$ J. K. Thomson, H. K. Wickramasinghe, and E. A. Ash, J. Phys. D 6, 677 (1973).

${ }^{17}$ K. P. Kostli and P. C. Beard, Appl. Opt. 42, 1899 (2003).

${ }^{18}$ J. D. Hamilton, T. Buma, M. Spisar, and M. O'Donnell, IEEE Trans. Ultrason. Ferroelectr. Freq. Control 47, 160 (2000).

${ }^{19}$ J. M. Cannata, T. A. Ritter, W. H. Chen, R. H. Silverman, and K. K. Shung, IEEE Trans. Ultrason. Ferroelectr. Freq. Control 50, 1458 (2003).

${ }^{20}$ K. Crain, Surf. Mount. Technol. 9, 50 (1995). 